\title{
Early Gastric Cancer Associated with Synchronous Liver Metastasis
}

\author{
KIKUO KOUFUJI, JINRYO TAKEDA, KEN HASHIMOTO, \\ TAKAHO TANAKA AND TERUO KAKEGAWA \\ Department of Surgery, Kurume University School of Medicine, \\ Kurume, 830 Japan
}

Received for publication July 24, 1991

\begin{abstract}
Summary: During the 14 years from 1976 to 1989,1442 gastric cancers were resected in the Department of Surgery at Kurume University. $546(37.9 \%)$ were primary early gastric cancers. One $(0.2 \%)$ of these was associated with a synchronous liver metastasis. In the advanced gastric cancers, the rate of synchronous liver metastasis was $12.8 \%$. However, synchronous liver metastasis with an early gastric cancer is quite rate. Only 19 cases have been reported in the Japanese literature. A case involving an early cancer, $10 \mathrm{~mm}$ in diameter, that was associated with liver metastasis is reported.
\end{abstract}

Key words: early gastric cancer-synchronous liver metastasis-cardiatotal gastrectomy - partial liver resection - intra-arterial infusion

\section{Introduction}

Early gastric cancer (EGC) is defined as a carcinoma confined to the mucosa (m-cancer) or submucosa (sm-cancer) of the stomach, with or without lymph node metastasis. The lymph node metatasis rate is accepted as $3-5 \%$ for m-cancer and about $20 \%$ for sm-cancer. However, the prognosis of EGC is generally good, with a 5-year survival rate of $95-98 \%$ for $\mathrm{m}$-cancer and $90-95 \%$ for sm-cancer. When lymph node metastasis is positive, it is mainly limited to Group 1 lymph nodes, which are treatable by the standard operation of gastrectomy with Group 1 and 2 lymph node dissection.

In the advanced gastric cancers, synchronous liver metastasis was $12.8 \%$ in a previous study (Takeda et al. 1990). On the other hand; liver metastasis in EGC was very low. Only 19 cases of EGC associated with synchronous liver metastasis have been reported, in the Japanese literature (Sugazawa et al. 1990; Habu et al. 1991).
A case of a small EGC, only $10 \mathrm{~mm}$ in diameter, in the gastric cardia associated with synchronous bilateral liver metastases is described.

\section{Patients and Methods}

During the 14 years from 1976 to 1989 , 1442 gastric cancers were resected in the First Department of Surgery, Kurume University Hospital. Of these, 546 (37.9 $\%$ ) were early gastric cancers. Only one $(0.2 \%)$ of these early gastric cancers, was associated with synchronous liver metastasis.

\section{Report of Case}

A sixty-two year old male patient was referred for gastric surgery to the First Department of Surgery, Kurume University Hospital. This patient had a gastric cancer which was confirmed histologically from preoperative biopsies. The small 
EGC of the superficial elevated type IIa were found in the upper third of the stomach, by double contrast gastro-duodenal X-ray examination. Detailed preoperative ultra-sonogram (US) examinations revealed a low density area, suspected to be tumor masses, in the right lobe of the liver. Figure 1 shows the two liver tumors on a CT-scan of the right lobe (black arrow) that were suspected to be metastases from the gastric cancer. This patient underwent a total gasrectomy with liver tumor resection and intra-proper hepatic arterial cannulation

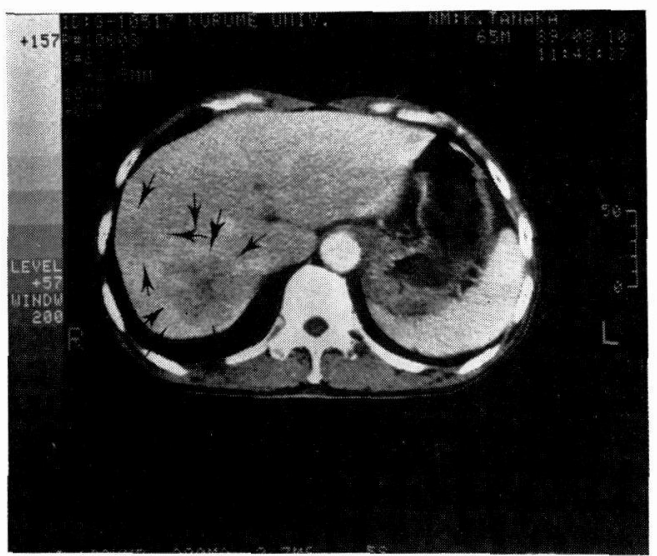

Fig. 1. The black arrows identify 2 liver tumors in the right lobe of the liver on a CT-scan.

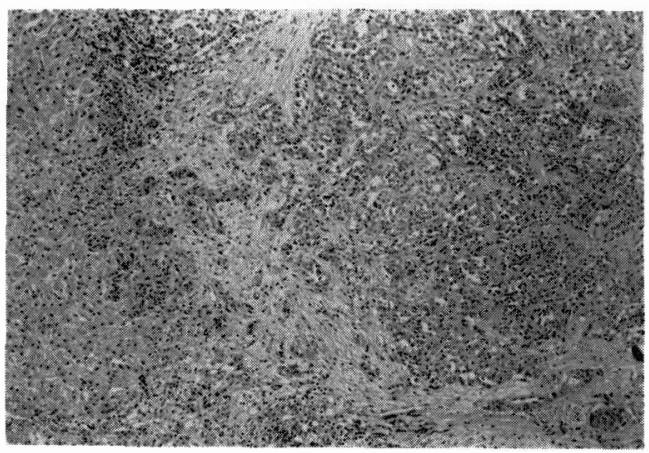

Fig. 3. A poorly differentiated adenocarcinoma in the liver. The normal liver cells can be seen on the left $(\mathrm{HE} \times 50)$. for postoperative local administration of anti-cancer drugs to both lobes. Figure 2 shows the resected gastric specimen. The gastric cancer was small, only $10 \mathrm{~mm}$ in diameter, and was located in the upper third of the stomach (black arrow). Pathologically, the cancer was a poorly-differentiated adenocarcinoma and with cancer depth within the submucosa of the stomach. Figure 3 is a hematoxin-eosin stained preparation of the liver tumor that confirmed the same histological type to be the same the primary gastric cancer. Figure 4 is a positively stained liver tu-

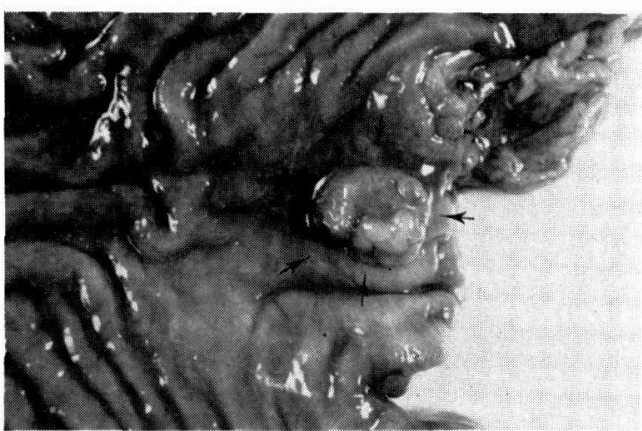

Fig. 2. A resected specimen of an early gastric cancer type $\Pi$ la (black arrow) just below the esophago-gastric junction.

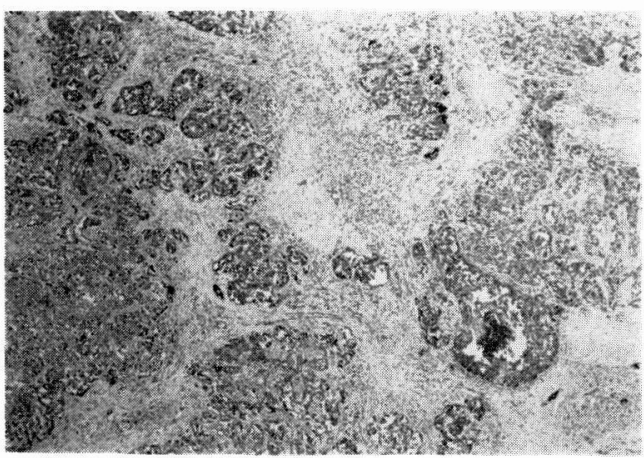

Fig. 4. A metastatic liver tumor positively stained by CA 19-9 histoimmunochemical methods $(\mathrm{ABC}$ method $\times 20)$. 
mor after CA19-9 staining. Moreover, liver tumor was negatively stained by AFP. Therefore, these liver tumors were diagnosed as associated synchronous liver metastasis.

No postoperative complication relating to the anastomosis occurred. However, the cannulated arterial catheter became blocked, 40 days postoperatively. This patient died of a liver metastasis with jaundice, 13 months postoperatively.

\section{Discussion}

The liver, by virtue of its location, blood supply, anatomy and other unknown factors, provides a major secondary location for metastases from any malignant tumors of the stomach. Gastric cancer associated with liver metastasis is common for advanced cancers. The rate of liver metastases in advanced gastric cancers was found to be $12.8 \%$ in a previous study (Takeda et al. 1990). On the other hand, for EGC, synchronous liver metastasis is rare. Only 19 cases have been reported in the Japanese literature. The incidence of EGC with synchronous liver metastases has been reported to be 0.2-1.6\% (Sugazawa et al. 1990; Habu et al. 1991). However, there is general agreement that hematogenous metastasis, especially liver metastasis, is frequent in cases with recurrent gastric cancers after resection of the EGC (Fujita et al. 1983).

A rare case of EGC, only $10 \mathrm{~mm}$ in diameter, associated with synchronous liver metastasis was described in this report with special attention to the metastatic routes from the stomach to the liver, and the surgical management.

This EGC was a sm-cancer with positive lymph node metastases in the pericardia and lymph nodes along the hepatoduodenal ligaments. Moreover, submucosal lymph vessels and venous vessels were involved. Therefore, the gastric cancer cells probably first detached from the mucosa and submucosa, and infiltrated to the submucosal lymph and venous vessels of the stomach. These cancer cells then detached again and infiltrated the regional lymph nodes and proliferated there. The metastasized cancer cells in the lymph nodes and infiltrated cancer cells in the lymph and/or venous vessels again detached and were transported through the portal system and/or direct lymph routes into the liver and then proliferated in both lobes of the liver.

The prognosis for gastric cancer with liver metastasis is quite boor-nearly all patients die within 2 years of the diagnosis. The prognosis for EGC associated with liver metastasis is thought to be as poor as that for advanced gastric cancer with liver metastasis. Gastric cancer with liver metastasis is considered to be inoperable and the treatment for most types has often been laparotomy, alone. The recent rapid progress in preoperative diagnosis on the extent of liver metastasis using US, CT, and MRI, and in postoperative care and intra- and lpostoperative chemotherapy have enabled a safe non-combined curative resection of the primary gastric cancer; which allows for improved chances of survival after a combined resection. There have been studies on the prognostic significance of the primary tumor resection for gastric cancer patients with liver metastases. In advanced cases, liver metastasis frequently occurs in gastric cancer patients together with peritoneal dissemination, juxta lymph node metastasis and cancer invasion to adjacent organ (s). However, in EGC, there is generally no peritoneal dissemination and no cancer invasion to an adjacent organ. Therefore, EGC with liver metastasis has a much better prognosis than advanced gastric cancer with liver metastasis. In the present study, 
gastric resection and lymph node dissection were possible during a combined resection of the left metastatic liver tumor. However, the tumors in the right lobe could not be resected. Arterial cannulation for infusion of anti-cancer drugs was then performed intraoperatively and the infusion was continued until occlusion occured.

Gastric cancer metastasis in the liver is rarely solitary. However, recent reports of prolonged survival can be found in the Japanese literature after primary gastric cancer resection with combined partial resection of the metastatic liver tumor, and with combined continuous intra-arterial infusion of anti-cancer drugs (Fujimoto et al. 1987). Such a long survival could not be obtained in the present case. However, it is important to not give up on gastric cancer patients, especially early gastric cancer with liver metastasis.

\section{References}

Fujimoto, S., Seresta, R.D., Kasanuki, J., Kokubun, M., Iсhікі, N., Miyazaki, A., Онта, M. and Окuг, K. (1987). Gastric cancer with synchronous unresectable hepatic metastasis and a positive response to chemotherapy. Jpn. J. Surg. 17, 307-311.
Fujita, Y., Nishioka, B., Sakita, M., Kojima, O., Nomiyama, S., Ouchi, T., Yamane, T., Kasuga, M. and Majima, S. (1983). Conservative surgery for regional lymphadenectomy in the treatment of early gastric carcinoma. Jpn. J. Surg. 13, 184-190.

Habu, H., Takeshita, K., Sunagawa, M. and Endo, M. (1986). Lymph node metastasis in early gastric cancer. Int. Surg. 71, 244-247.

Japanese Research Society for Gastric Cancer (1981). The general rules for the gastric cancer study in surgery and pathology. Jpn. J. Surg. 11, 127-139.

Koga, S., Takebayashi, M., Kaibara, N., Nishidoi, H., Kimura, O., Kawasumi,H. and Makino, M. (1987). Pathological characteristics of gastric cancer that develop hematogenous recurrence, with special reference to the site of recurrence. J. Surg. Oncol. 36, 239-242.

OkuYama, K., Onoda, S., Tohnosu, N., Yamamoto, Y., Hanaoka, A., Koide, Y., Seki, S., Hara, T. and Isono, K. (1988). The prognostic significance of resection of primary tumor in gastric and colorectal cancer patients with synchronous liver metastasis. Jpn. J. Surg. 18, 7-17.

Sugazawa, A., Yamane, Y., Kawano, K., Maki, A., Abe, S. and UedA, U. (1990). A case report of early gastric cancer with multiple liver metastasis. Rinshogeka 45, 123-127.

(in Japanese)

Takeda, J., Yasumoto, K., Yano, S., Tanaka, T., Koufuji, K., Hashimoto, K. and Kakegawa, T. (1990). Retrospective studies of gastric cancer with hepatic metastasis. Kurume Med. J. 37, 271-276. 